\title{
The eccentricity-mass distribution of exoplanets: signatures of different formation mechanisms?
}

\author{
I. Ribas ${ }^{1}$ and J. Miralda-Escudé ${ }^{1,2}$ \\ ${ }^{1}$ Institut de Ciències de l'Espai (CSIC-IEEC), Campus UAB, Facultat de Ciències, Torre C5, parell, 2a planta, \\ 08193 Bellaterra, Spain \\ e-mail: [iribas; miralda]@ieec.uab.es \\ 2 Institució Catalana de Recerca i Estudis Avançats, Barcelona, Spain
}

Received 31 May 2006 / Accepted 6 December 2006

ABSTRACT

\begin{abstract}
We examine the distributions of eccentricity and host star metallicity of exoplanets as a function of their mass. Planets with $M$ sin $i \gtrsim$ $4 M_{\mathrm{J}}$ have an eccentricity distribution consistent with that of binary stars, while planets with $M \sin i \lessgtr 4 M_{\mathrm{J}}$ are less eccentric than binary stars and more massive planets. In addition, host star metallicities decrease with planet mass. The statistical significance of both of these trends is only marginal with the present sample of exoplanets. To account for these trends, we hypothesize that there are two populations of gaseous planets: the low-mass population forms by gas accretion onto a rock-ice core in a circumstellar disk and is more abundant at high metallicities, and the high-mass population forms directly by fragmentation of a pre-stellar cloud. Planets of the first population form in initially circular orbits and grow their eccentricities later, and may have a mass upper limit from the total mass of the disk that can be accreted by the core. The second population may have a mass lower limit resulting from opacity-limited fragmentation. This would roughly divide the two populations in mass, although they would likely overlap over some mass range. If most objects in the second population form before the pre-stellar cloud becomes highly opaque, they would have to be initially located in orbits larger than $\sim 30 \mathrm{AU}$, and would need to migrate to the much smaller orbits in which they are observed. The higher mean orbital eccentricity of the second population might be caused by the larger required intervals of radial migration, and the brown dwarf desert might be due to the inability of high-mass brown dwarfs to migrate inwards sufficiently in radius.
\end{abstract}

Key words. planetary systems - planetary systems: formation - binaries: general - stars: low-mass, brown dwarfs - stars: formation

\section{Introduction}

The discovery of the first exoplanets around main-sequence stars a decade ago (Mayor \& Queloz 1995; Butler \& Marcy 1996; Marcy \& Butler 1996; Butler et al. 1997) has provided us with a first glimpse at the fascinating diversity of planetary systems in the universe. At present, with about 200 exoplanets known (most of them having been found by the method of radial velocities around stars similar to the Sun, and a few by the methods of transits and microlensing events), we have learned that: (a) Jovian planets are found over a wide range of orbital radii $a$, from the smallest orbits where they can survive evaporation $(a \sim 0.03 \mathrm{AU})$ to the largest ones at which they can be detected ( $a \sim 5 \mathrm{AU})$; (b) Jovian planets that have not been tidally circularized show a wide range of eccentricities, with a median value of $\sim 0.3$; (c) orbital resonances are commonly found in systems when more than one planet is detected; (d) there is a power-law distribution of planet masses that is roughly uniform in $\log M$ from $\sim 0.3$ to $\sim 10$ Jupiter masses (e.g., Tabachnik \& Tremaine 2002), although with a strong deficit of objects with mass greater than $\sim 0.015 M_{\odot}$ (designated as brown dwarfs), which is known as the "brown dwarf desert", and probably an increase in the planet abundance at lower masses, as suggested by the recent detections of Neptune-like planets with the method of microlensing (Beaulieu et al. 2006; Gould et al. 2006). All of these findings have come as surprises, and none of them was predicted or expected from theories of planetary formation.

With the increasing sample of exoplanets available for statistical studies, the fundamental question of the planet formation mechanism and their subsequent orbital evolution that results in the observed distribution may start to be addressed. The only information we have so far that relates to the formation process of planets is the distribution of their orbital periods, eccentricities, and planet masses, which can also be compared to the same distributions for binary stars and brown dwarfs. In addition, properties of the host stars such as their metallicity can be included. Jovian planets are thought to form in circumstellar disks from the coalescence of planetesimals and gravitational accretion of gaseous material (Pollack et al. 1996). This process should lead to orbits that are initially circular, but they could subsequently be perturbed by several mechanisms causing an increase of the eccentricity, such as disk-planet interactions (Kley \& Dirksen 2006), the Kozai mechanism (Kozai 1962; Holman et al. 1997; Takeda \& Rasio 2005), and close encounters or resonant interactions between planets (Chiang et al. 2002; Ford et al. 2005; see also the review on eccentricity growth mechanisms by Tremaine \& Zakamska 2004). These mechanisms need to be effective for a large majority of planets formed in disks since most of the detected exoplanets are found to possess eccentricities much larger than solar system planets (and hence, the solar system must be an oddity among planetary systems).

It has been noted before that the distribution of eccentricities of the exoplanets seems to be remarkably similar to that of binary systems (Heacox 1999; Stepinski \& Black 2000, 2001), with a slight tendency for the eccentricities to increase with planet mass (see Tremaine \& Zakamska 2004; Marcy et al. 2005; Papaloizou \& Terquem 2006). This result is surprising because 
it is not clear how a dynamical process can result in higher eccentricities acquired by more massive planets. Although interactions with a gaseous disk can generate eccentricities more easily for the most massive planets, it is doubtful that this process alone can excite the eccentricities up to the observed distribution. While the Kozai mechanism predicts an eccentricity distribution independent of planetary mass, perturbations among planets would likely tend to leave lower mass planets with higher eccentricities, basically from energy equipartition arguments; it is possible, however, that if one observes the most massive planet that has survived in every planetary system, the systems with greater total mass compared to the host star have been more strongly perturbed.

Contrary to planetary objects, the most favored hypothesis to explain the formation of binary stars and brown dwarf companions is by fragmentation of the parent molecular cloud during the gravitational collapse process, or as a result of gravitational instability or fission of a rapidly rotating pre-stellar cloud (Tohline 2002). If the fragmentation process resulted in an orbit randomly selected from phase space for a fixed orbital energy, the distribution of eccentricities should be uniform in $e^{2}$, with a median eccentricity of 0.7 in a binary star sample. However, this is not observed (e.g., Abt 2005), and even after removing systems that may have been affected by tidal circularization the typical eccentricities are substantially lower. It is particularly striking that the distribution of eccentricities of exoplanets and binary stars are remarkably similar, since the two types of systems are thought to form by different mechanisms.

In this paper, we consider the possibility that some planetary mass objects have also formed by the process of direct cloud fragmentation. The known exoplanets would therefore be part of two populations (as proposed earlier by, e.g., Black 1997; Mayor et al. 1998; Papaloizou \& Terquem 2001; Udry et al. 2002), stemming from two different mechanisms of mass growth: either gas accretion from a disk onto a seed planetesimal, or fragmentation during the collapse of the gas cloud. Even though exoplanets and brown dwarfs are usually considered as separate classes of objects (with the separation chosen at a mass of $0.013 M_{\odot}$, the minimum mass required for deuterium burning), there is no fundamental reason why these objects should belong to distinct classes from the point of view of their formation. In general, the different formation processes mentioned above (as well as disk fragmentation by gravitational instability) may be relevant for objects over wide ranges of mass that may overlap, and should be unrelated to the minimum mass for deuterium burning or for hydrogen burning. We shall search for possible signatures of the presence of two populations of objects among the known exoplanets and brown dwarfs with the improved statistics available today, and also examine the correlation with the metallicity of the host star.

\section{Eccentricity distribution of exoplanets and binary stars}

We start by examining the distribution of eccentricity and $M \sin i$ in the sample of known exoplanets and brown dwarf companions within $5 \mathrm{AU}$ of their star. We use the sample of all known exoplanets around normal stars with measured orbital elements from radial velocities (see, e.g., the Extrasolar Planets Encyclopaedia; Butler et al. 2006). We add to these a set of 17 objects with $0.013 M_{\odot}<M \sin i<0.08 M_{\odot}$, which are within $5 \mathrm{AU}$ of a star of F, G, or K spectral type (note that more brown dwarfs are known at this distance from $\mathrm{M}$ dwarfs, where they seem to

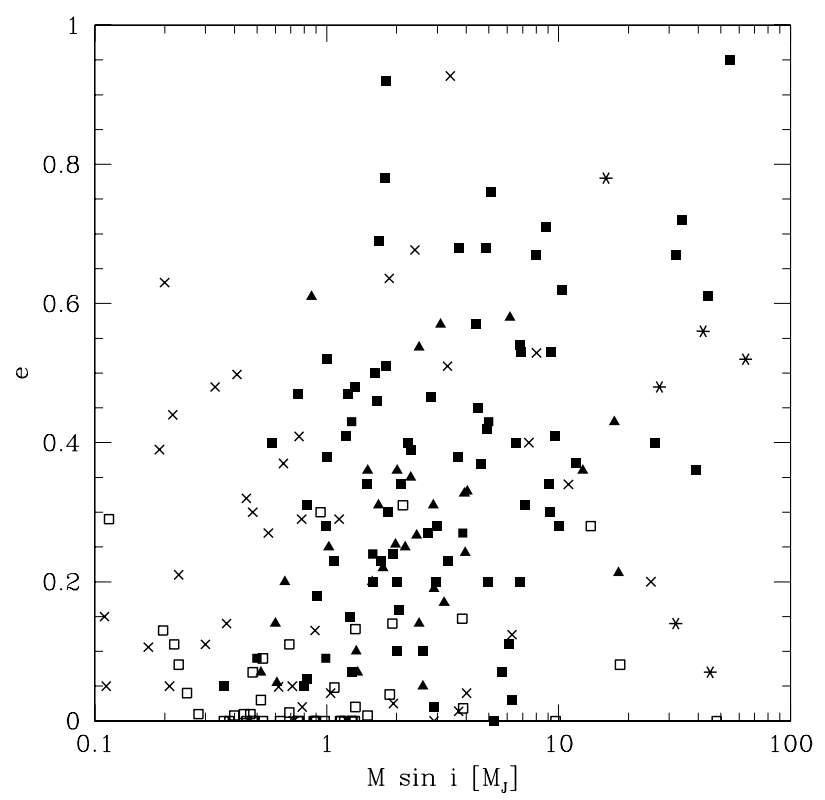

Fig. 1. Eccentricity versus mass for the sample of substellar objects. Open squares: planets with $a<0.1 \mathrm{AU}$. Crosses: planets with $0.1 \mathrm{AU}<$ $a<0.5$ AU. Filled squares: planets with $a>0.5$ AU. Triangles: planets with $a>0.5 \mathrm{AU}$ in systems with more than one detected planet. Stars: objects that have been found to have a stellar mass from Hipparcos astrometric observations.

be more common; see Close et al. 2003). This forms our sample of "substellar objects", a total of 204. Of course, many of the 17 objects with $M \sin i>0.013 M_{\odot}$ may actually be stars. Because of the presence of the brown dwarf desert (a dearth of brown dwarfs orbiting within $5 \mathrm{AU}$ of a solar-type star compared to either stellar or planetary-mass companions), an object found with $M \sin i$ in the range corresponding to a brown dwarf mass is likely to be a star with a small orbital inclination. In fact, 6 of these 17 objects (HD 112758, HD 110833, HD 169822, HD 217580, HD 18445, and BD-04 782) have measured astrometric orbits by Hipparcos that confidently place their masses in the stellar regime (as long as the error bars are correct; see Halbwachs et al. 2000; Vogt et al. 2002), another 3 are uncertain (HD 283750, HD 114762, and HD 140913; Halbwachs et al. 2000), and the other 8 are likely to be true brown dwarfs (HD 180777, HD 89707, HD 137510, HD 127506, HD 184860 , HD 202206b, HD 168443c, and HD 29587; see Halbwachs et al. 2000; Vogt et al. 2002; Endl et al. 2004; Galland et al. 2006). Although a brown dwarf desert is clearly present, it is not completely empty.

We show the distribution of eccentricity and $M \sin i$ for all the objects in our sample, 204 objects in total, in Fig. 1 (10 of the planets have $M \sin i<0.1 M_{\mathrm{J}}$ and do not appear in the figure). We have plotted them with different symbols depending on their semimajor axis. Objects with $a<0.1$ AU have likely been affected by tidal circularization, and clearly have small eccentricities. Some of the objects in the middle group could have been affected by tidal circularization, especially for $a<0.2 \mathrm{AU}$ and very high eccentricities, and in cases where the planets remained larger than their present size for a substantial time in their youth. There is, however, no clear tendency for objects with $0.1 \mathrm{AU}<a<0.5 \mathrm{AU}$ to show smaller eccentricities than objects with $a>0.5 \mathrm{AU}$ at similar values of $M \sin i$. Objects that are also at $a>0.5 \mathrm{AU}$ and belong to systems with more than one planet detected do not have an obviously different distribution, suggesting that being part of a planetary system does not greatly affect 


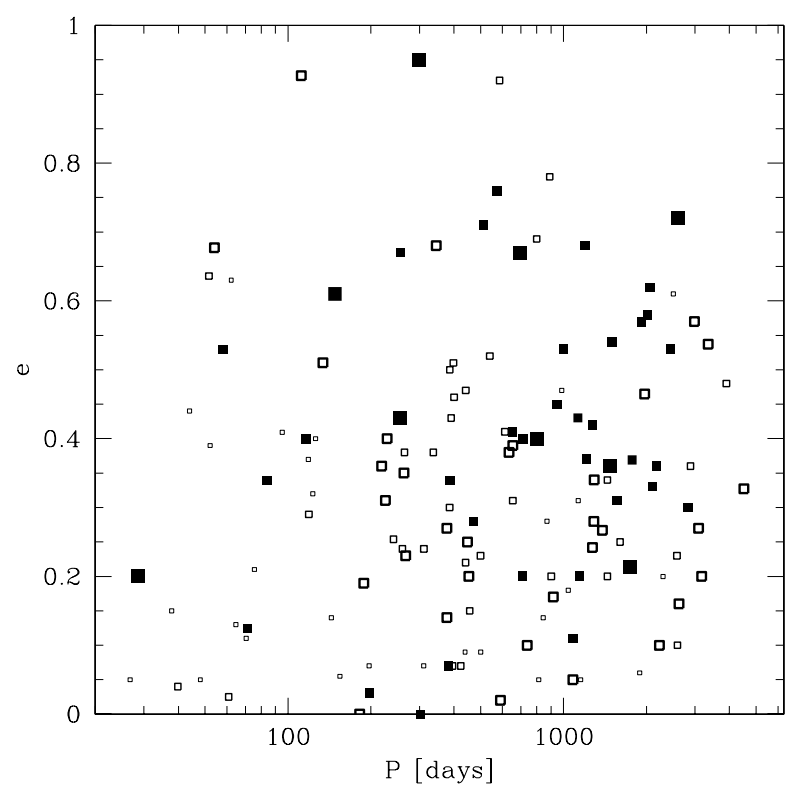

Fig. 2. Eccentricity versus orbital period for all planets and brown dwarfs of different mass groups; objects with $a<0.2$ AU have been removed from the sample. Small open squares: $M \sin i<M_{\mathrm{J}} ;$ medium open squares: $M_{\mathrm{J}}<M \sin i<2 M_{\mathrm{J}}$; large open squares: $2 M_{\mathrm{J}}<$ $M \sin i<4 M_{\mathrm{J}}$; large filled squares: $4 M_{\mathrm{J}}<M \sin i<13 M_{\mathrm{J}}$; extralarge filled squares: $13 M_{\mathrm{J}}<M \sin i$.

the eccentricity. Finally, six objects with $M \sin i>0.013 M_{\odot}$ are stars with low orbital inclinations according to Hipparcos measurements.

One can also appreciate from Fig. 1 that there is a tendency for the orbital eccentricity to increase with mass, as mentioned in the introduction. The effect is, however, a weak one (we have included the two recently discovered planets by Jones et al. 2006, which go against this mean tendency). There is a natural concern that this correlation might be induced by observational selection effects: according to Cumming (2004), high eccentricities tend to be more easily detectable for long period orbits, and low eccentricities are easier to detect for short periods. Because low-mass planets are harder to detect for long periods (because of the low velocity amplitude), a change in the eccentricity distribution with orbital period due to selection bias might induce the observed dependence of eccentricities with planet mass. To check for this possibility, in Fig. 2 we plot the eccentricity versus the period for the sample of all the exoplanets and brown dwarfs that have $a>0.2$ AU (to remove the objects affected by tidal circularization), using different symbols for different ranges of $M \sin i$ (the largest points correspond to more massive planets). While one can also discern in Fig. 2 the tendency for eccentricity to increase with mass, there is no obvious variation of eccentricity with period for planets of fixed mass. We shall assume in this paper that the mass-eccentricity relation is not being severely affected by selection effects, although this will require more careful examination as the number of known exoplanets increases.

We also use the sample of binary stars based on The 9th Catalog of Spectroscopic Binary Orbits (Pourbaix et al. 2004). From this catalog we selected binary stars according to several criteria: (a) a quality flag of 2 or greater to ensure reasonably firm orbital solutions and reliable eccentricities (tests using different selections in the quality flag indicate that this does not introduce any bias in the eccentricity distribution); (b) the orbital period is required to be larger than 30 days, large enough so that circularization processes have not played an important role;

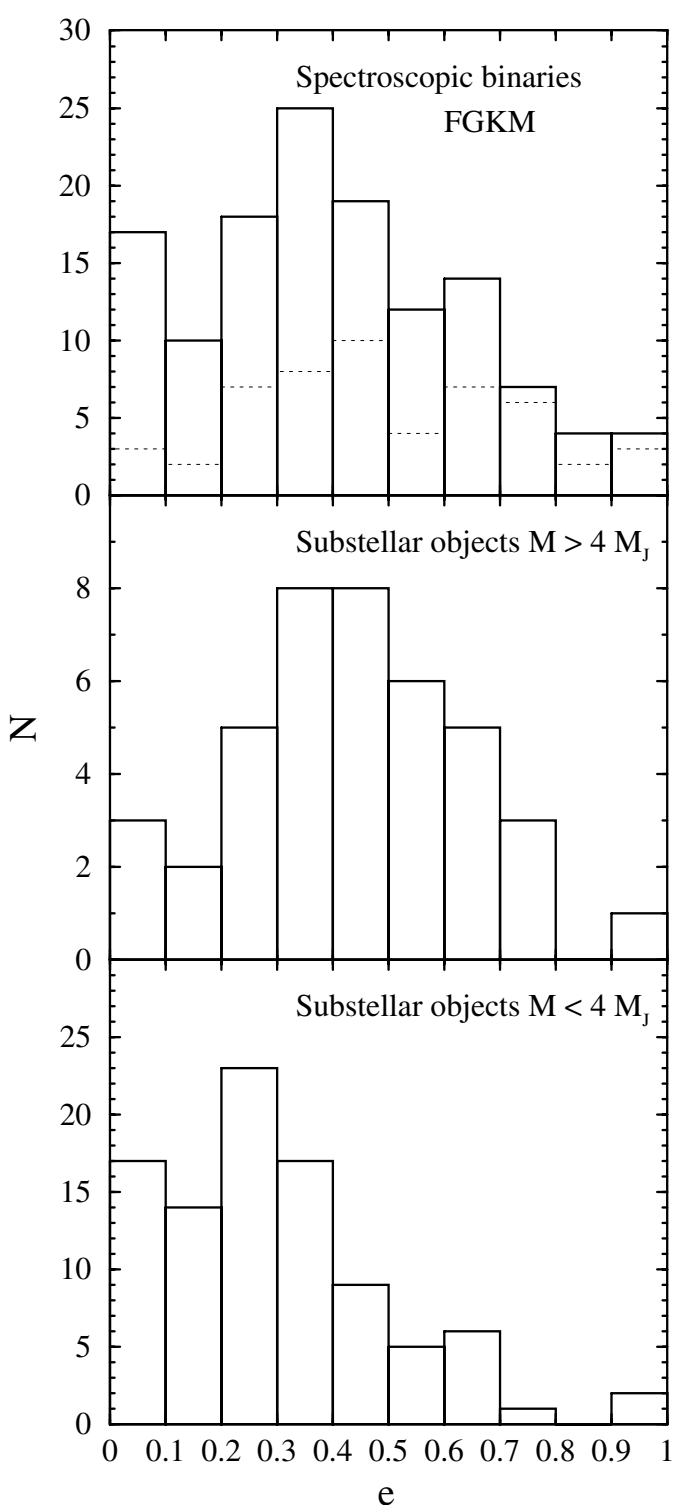

Fig. 3. Eccentricity distributions for the three samples considered in this work: spectroscopic binaries (top), substellar objects with $M>4 M_{\mathrm{J}}$ (middle), and substellar objects with $M<4 M_{\mathrm{J}}$ (bottom). All objects with $a<0.2$ AU have been removed from the sample to avoid the effects of tidal circularization. The histogram represented with a dotted line in the top panel corresponds to double-lined spectroscopic binaries only (see text for discussion).

(c) only main-sequence or subgiant components are kept to minimize the range of stellar radii and permit the use of the orbital period as a measure of the significance of circularization processes. Similar criteria were recently used by Abt (2005) to study the eccentricity distribution of binary stars. The resulting sample is composed of 200 spectroscopic binaries meeting the restrictions described above. In addition, we consider the subsample of these 200 binaries having star components of FGKM spectral type, for similarity with exoplanet host stars. This subsample is composed of 130 spectroscopic binaries.

The eccentricity distributions of the samples considered in this work are shown as histograms in Fig. 3. The top panel is for the spectroscopic binaries with components of FGKM spectral types. The middle and bottom panels show the eccentricity distributions of substellar objects with minimum masses above and below $4 M_{\mathrm{J}}$, respectively (the middle panel includes the 
Table 1. Results of the Kolmogorov-Smirnov tests.

\begin{tabular}{ccccc}
\hline \hline Distrib \#1 & Distrib \#2 & $D_{\text {obs }}$ & $p\left(D>D_{\text {obs }}\right)$ & $n_{\text {eff }}$ \\
\hline$M \sin i<4 M_{\mathrm{J}}$ & $M \sin i>4 M_{\mathrm{J}}$ & 0.359 & 0.001 & 28 \\
SB FGKM & $M \sin i<4 M_{\mathrm{J}}$ & 0.251 & 0.002 & 54 \\
SB FGKM & $M \sin i>4 M_{\mathrm{J}}$ & 0.125 & 0.682 & 31 \\
SB all & $M \sin i<4 M_{\mathrm{J}}$ & 0.238 & 0.001 & 63 \\
SB all & $M \sin i>4 M_{\mathrm{J}}$ & 0.150 & 0.400 & 34 \\
\hline
\end{tabular}

8 objects with $13 M_{\mathrm{J}}<M \sin i<80 M_{\mathrm{J}}$ that we consider to be likely brown dwarf companions, as discussed above). The placing of the mass division at $4 M_{\mathrm{J}}$ is somewhat arbitrary at this point, although there are observational indications of a change in planet properties around this value (Udry et al. 2002). Further discussion on the mass limit is provided in Sect. 4. All substellar objects with $a<0.2 \mathrm{AU}$ have been eliminated from the sample to remove any orbits that have been affected by tidal circularization.

To compare the distributions we made use of the Kolmogorov-Smirnov (K-S) two-sample test following the implementation of Press et al. (1992). The tests were carried out with the three samples described above. Given two cumulative distribution functions $S_{1}(x)$ and $S_{2}(x)$ with $n_{1}$ and $n_{2}$ data points, respectively, the K-S statistic is defined as $D=$ $\max \left|S_{1}(x)-S_{2}(x)\right|$ and the effective number of data points is $n_{\text {eff }}=\left(n_{1} n_{2}\right) /\left(n_{1}+n_{2}\right)$. Then the probability that the real value of $D$ is greater than its observed value $D_{\text {obs }}, p\left(D>D_{\text {obs }}\right)$, yields an estimate of the likelihood of the null hypothesis that the two distributions have been drawn from the same population.

The results of the K-S tests are presented in Table 1. The sample of spectroscopic binaries and high-mass planets clearly have eccentricity distributions consistent with being identical. However, the eccentricity distribution of the low-mass planets is found to be different. The probability for the distributions to be the same is around $0.1 \%$ to $0.2 \%$. The main difference in the distributions is that low-mass planets tend to have lower eccentricities, as shown in Fig. 3.

\subsection{Binary stars on nearly circular orbits}

There is an additional surprising feature in the eccentricity distribution of the spectroscopic binaries: the excess of binaries with very low eccentricities, $e<0.1$, compared to a distribution that grows linearly with $e$ for small $e$, as expected if all orbits have been subject to a similar dynamical evolution inducing random variations in their eccentricity, which should uniformly fill the available phase space at small $e$. We have checked that this excess is not the result of systems with unreliable observations assumed to have a circular orbit, and it does not appear to be due to any other observational bias.

To further investigate this excess of low-eccentricity orbits, the dotted histogram in the top panel of Fig. 3 shows the distribution of double-lined spectroscopic binaries only. Doublelined binaries generally have components of similar mass. Very roughly, a system in which the ratio of luminosity of the two components is above $0.1-0.05$ will permit the identification of both lines in the spectra, and this ratio of luminosities corresponds to a mass ratio $\sim 0.5$ for main-sequence components. We can see that the excess of low-eccentricity orbits is more pronounced in systems with unequal components.

This feature in the eccentricity distribution suggests that a small fraction $(\sim 10 \%)$ of the binaries are formed on nearly circular orbits and experience very little orbital evolution

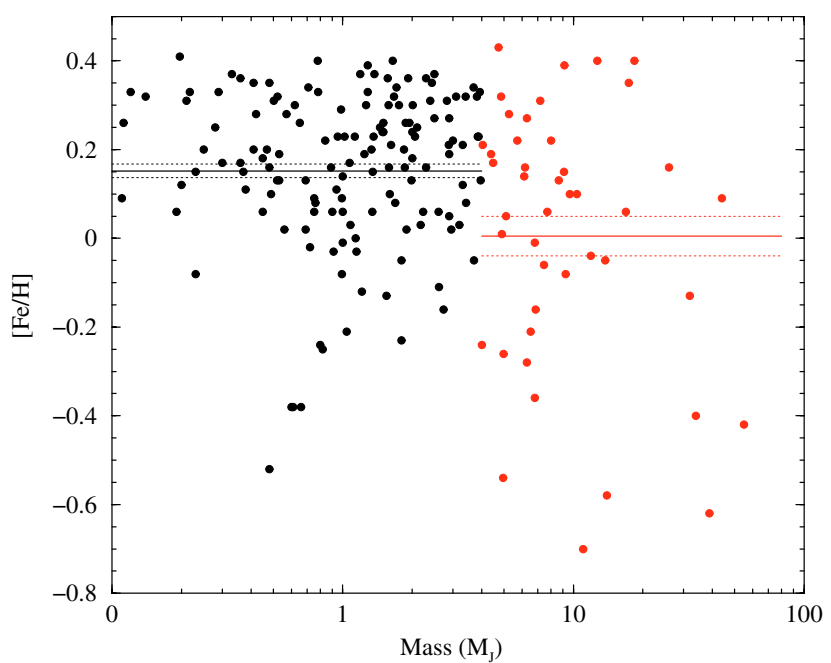

Fig. 4. Mass versus metallicity for the substellar objects in the sample. Mean metallicity values for the two proposed populations (minimum mass above and below $4 M_{\mathrm{J}}$ ) are represented by the horizontal lines (with the corresponding error bars shown as dotted lines).

afterwards. High-mass planets might also have this excess of orbits at small eccentricities, although their numbers are at present too small to make this statistically significant. Low-mass planets do not seem to show any excess of low-eccentricity orbits.

\section{Host star metallicity distribution of exoplanets}

Another property of exoplanets that can inform us about their formation mechanism is the metallicity of the host star. The probability that a star hosts planets of the type detected in radial velocity surveys increases rapidly with stellar metallicity (e.g., Fischer \& Valenti 2005). The implication is that the formation of Jovian planets is made more likely when heavy elements have a high abundance (the possibility that stellar metallicities at the photosphere are increased by accreted planets was discarded by examining the dependence of the metallicity with the spectral type of the star; see Pinsonneault et al. 2001). Our examination of the eccentricity-planet mass correlation in Sect. 2 suggested that planets may be divided into two populations, roughly those more and less massive than $\sim 4 M_{\mathrm{J}}$. It is therefore worthy to see if the metallicity of the host star shows any similar change around a comparable mass.

Figure 4 shows the metallicities of all stars hosting planets, collected from the literature, as a function of the planet mass. The average host star metallicity of planets with $M \sin i<4 M_{\mathrm{J}}$ and $M \sin i>4 M_{\mathrm{J}}$ is indicated as is the error of the average metallicity. The average metallicity of stars with planets of mass below $4 M_{\mathrm{J}}$ is $[\mathrm{Fe} / \mathrm{H}]=0.152 \pm 0.015$, while stars with substellar objects above that mass have a mean metallicity of $[\mathrm{Fe} / \mathrm{H}]=$ $0.005 \pm 0.045$. The difference is therefore at the 3- $\sigma$ level. For comparison, the average metallicity in the solar neighborhood is $[\mathrm{Fe} / \mathrm{H}] \simeq-0.15$ with a scatter of $\sim 0.2 \mathrm{dex}$ (Nordström et al. 2004; Fischer \& Valenti 2005; Valenti \& Fischer 2005). The population of stars with massive substellar objects probably has a metallicity value consistent with that of the field, especially when considering that the sample of stars for which radial velocity searches have been made is biased to high metallicity. Visual examination of the dots suggests that the tendency of the host star metallicity to decrease with planet mass is a gradual one, over the range from $\sim 1$ to $50 M_{\mathrm{J}}$. 


\section{Discussion}

\subsection{A hypothesis for the physical origin of two planet populations}

The statistical evidence for the two trends we have examined, of increasing orbital eccentricity and decreasing host star metallicity with planet mass, should be considered as marginal at this point. However, the presence of the two independent trends reinforce each other in suggesting that there may be different planetary formation mechanisms that give rise to planets of different masses. In this section, we discuss a possible physical explanation for these trends, assuming that they are correct and bearing in mind that they will need to be confirmed (or refuted) by improved statistical evidence as the number of known exoplanets increases.

The metallicity of the host star should not be affected by the fact that a planet has formed and has stayed in orbit around the star. Therefore, it seems reasonable to conclude that the majority of the high-mass planets form in a way that is less influenced by the metallicity than low-mass planets. The most natural interpretation is that many of the high-mass planets form through the same mechanism postulated for brown dwarfs, by direct fragmentation of the pre-stellar cloud (possibly combined with the interruption of gas accretion by radiation feedback from nearby stars) or of a circumstellar gaseous disk that is gravitationally unstable (e.g., Whitworth et al. 2006), while the low-mass planets form by the initial coagulation of a core of rock and ice and subsequent gas accretion onto the core (Pollack et al. 1996). There is indeed no reason to suppose that substellar objects formed by these two mechanisms would not overlap in mass. The formation of a planet from a core of rock and ice has a natural mass upper limit determined by the total mass that can be accreted from a circumstellar disk, at the time and the radius where the planetary core reaches a critical mass allowing gas accretion to start. At the same time, a natural lower limit to the mass of objects formed by fragmentation and direct gravitational collapse of gas is the opacity limit, which assumes that fragmentation will not be efficient once a pre-stellar cloud becomes opaque to its own cooling radiation, and is given by (Rees 1976):

$M_{\mathrm{op}}=M_{\mathrm{Ch}} \mu^{-9 / 4} f^{-1 / 2}\left(\frac{k T}{m_{\mathrm{p}} c^{2}}\right)^{1 / 4}$,

where $M_{\mathrm{Ch}}$ is the Chandrasekhar mass, $\mu$ is the mean molecular weight in units of the proton mass, $f$ is the emissivity of the cloud at the moment it becomes opaque (expressed as the fraction of the blackbody radiation that is emitted), and $T \sim 10 \mathrm{~K}$ is the cloud temperature. This opacity limit mass is likely to represent the smallest possible mass of an object formed by direct gravitational collapse of gas: even if fragmentation is still possible by gravitational instability in a rapidly rotating, highly opaque disk, it is unlikely that the gas temperature in a circumstellar disk around a young star would be low enough to bring the Jeans mass below the opacity limit value in the conditions of the protostellar nebula. The opacity limit mass is close to a Jupiter mass, suggesting that objects formed by gas fragmentation may extend into the high-mass planet regime and be found orbiting other stars with an abundance that is independent of the host star metallicity. On the other hand, gas planets formed by core accretion may not usually grow to more than a few Jupiter masses, form preferentially around metal-rich host stars starting from nearly circular orbits, and grow their orbital eccentricities to an average value lower than the more massive objects formed by fragmentation.

\subsection{Orbital migration of the high-mass population}

However, this simple idea cannot account for the observations without including an additional ingredient. If the lower limit to the mass of substellar objects formed by fragmentation of gas clouds is to have anything to do with the opacity limit, then these objects should form at very large distances from their stars because the density at which opacity sets in is $n_{\mathrm{H}} \sim 10^{10} \mathrm{~cm}^{-3}$ (e.g., Whitworth et al. 2006), and the size of a region containing the mass in Eq. (1) at this density is $~ 30$ AU. Therefore, these objects would have to be formed at large distances and then migrate to the much smaller orbits at which the known exoplanets have been detected.

It may not be unreasonable for the population of high-mass planets to have undergone a large radial migration. After all, we know that it is necessary for most of the planets to have experienced radial migration because it is believed that they can form only at radii large enough to allow for the presence of ice particles, and many planets are found within the ice-line. If planets always start on circular orbits when forming from a rock-ice core, their eccentricities would have to increase as they experience radial migration to account for the observed high eccentricities. The need for a large interval of radial migration for objects formed by fragmentation might then be the reason behind the increasing eccentricity with mass: while most of the highmass planets would have migrated over large intervals, some of the low-mass planets, which could form from solid cores in the protoplanetary disk at distances much closer than the high-mass population, may have migrated over radial intervals that are too small to grow the eccentricity by the same amount.

Possible mechanisms for the migration of planets formed at large distances include the dynamical interaction with lowmass planets formed in the circumstellar disk, a hydrodynamic and/or gravitational interaction with the gaseous circumstellar disk, or interaction with a disk of planetesimals. The orbit of a distant, high-mass planet could be perturbed to a small pericenter by external torques, interactions with other distant planets, or a Kozai mechanism, and then its semimajor axis could be greatly reduced by exchanging energy with planets formed at short distances, or by dissipative hydrodynamic or gravitational interactions with the disk.

If brown dwarfs and high-mass planets in orbit around solartype stars are indeed formed only at large distances, this might account also for the presence of the brown dwarf desert (note that brown dwarfs are abundant around M-type stars at typical distances of $\sim 1 \mathrm{AU}$, where they have probably formed by a mechanism similar to binary stars; Close et al. 2003). As the brown dwarf mass increases, a more massive disk needs to be present around the star in order that a sufficient amount of angular momentum can be absorbed by the disk to allow for the migration of the brown dwarf. It is possible that planets or brown dwarfs above some critical mass are not typically able to migrate to within a few AU of their host star, because their angular momentum is too large to be exchanged with the circumstellar disk.

Clearly, a model where high-mass planets and brown dwarfs form only at large distances predicts that many of these objects should still be found at late times in orbits of large radius around stars. If $\sim 1 \%$ of solar-type stars contain high-mass planets within $5 \mathrm{AU}$, then a similar number of stars should contain the same planets and brown dwarfs at distances $>30 \mathrm{AU}$, even if the migration process of these distant planets can be highly efficient (that is, a high fraction of the distant planets end up in orbits within $5 \mathrm{AU}$ ). In general, brown dwarfs and planets with 
$M>5 M_{\mathrm{J}}$ are abundant in young clusters, accounting for $\sim 10 \%$ of all the objects (see González-García et al. 2006, although in some cases the abundance may be lower; see Lyo et al. 2006). These same objects in young clusters are found only rarely as companions to solar-type stars on distant orbits (in $\sim 1 \%$ of the stars; R. Rebolo, priv. communication), although the abundance of these companions could be lower in clusters containing many stars compared to the field, because of tidal destruction of the binary systems. The case of the star $\epsilon$ Indi is noteworthy: at a distance of 3.6 parsecs, it is the 20th nearest star to the Sun and it has a pair of brown dwarfs at a separation of $1500 \mathrm{AU}$ that was discovered only very recently (McCaughrean et al. 2004). In summary, it seems plausible that there is a large enough reservoir of high-mass planets and brown dwarfs formed at large distances, some of which may undergo radial migration and account for the high-mass planets found within $5 \mathrm{AU}$ of their stars. The alternative possibility to the migration scenario would be that the high-mass planets can form at a radius near their final orbit at which they are observed, by direct gas collapse through gravitational instability of a rotating, opaque disk. However, if this mode of formation is to account for both binary stars and high-mass planets, the presence of the brown dwarf desert has no clear explanation.

\subsection{The eccentricity distribution of planets and binary stars}

A possible test for the idea that the large eccentricities of the high-mass planet population originate in a large interval of radial migration is related to the fact that all planets on small orbits should have migrated by large intervals, because planets formed by the core accretion process are thought to have formed in orbits outside the ice-line, so they can reach small orbits only by migrating. Therefore, at a small semimajor axis the eccentricity distribution should become more alike for low- and high-mass planets. However, it is hard to tell from Fig. 2 if there is any tendency of increasing eccentricity with decreasing period, and as mentioned in Sect. 2 any such dependence might be influenced by selection effects arising from the methods by which planets are found in Doppler surveys.

The hypothesis we have examined for the origin of the population of high-mass planets provides no satisfying explanation for why the eccentricity distributions of spectroscopic binaries and high-mass planets are so similar. Clearly, binary stars would have even greater difficulty for migrating inwards from a large orbit than brown dwarf companions. Binary stars are likely to form on an orbit of similar size to their final one, perhaps by gravitational instabilities in massive circumstellar disks or fission of a rapidly rotating pre-stellar cloud (see Tohline 2002, for a review of binary formation theories). In any case, it is clear that binary stars must form by very different mechanisms than any high-mass planets collapsing directly out of gas. Even if the high-mass planets formed on orbits of similar size as the binary stars, the need to account for the brown dwarf desert strongly suggests totally different formation mechanisms. Binary stars are likely to form in different ways in any case, in view of the wide range of orbital sizes, the known excess of twin binaries (with very similar component masses; Pinsonneault \& Stanek 2006), and the excess of small eccentricity orbits we mentioned at the end of Sect. 2. The similar eccentricity distributions of binary stars and high-mass planets may more likely be related to processes that occur after their formation; for example, migration of a high-mass planet through a disk and the formation of a star from gravitational instability in the disk might give rise to similar eccentricity distributions at the end of the process. In addition, a series of perturbations that change orbital eccentricities in a random way may typically produce a characteristic eccentricity distribution (see Juric \& Tremaine 2006).

\section{Summary}

We have found that the known exoplanets exhibit two trends in their properties that, although statistically marginal at this point, may be indicative of the presence of more than one population formed by different mechanisms: (a) the mean orbital eccentricity tends to increase with planet mass; and (b) the metallicity of the host star tends to decrease with planet mass. We also find that (c) the eccentricity distributions of planets more massive than a few Jupiter masses and of spectroscopic binaries are remarkably similar. Other known relevant facts for understanding the origin of exoplanets include the following: (d) many exoplanets must have migrated over large radial intervals to reach their present orbits from the location where they could form; (e) their final orbits must have been left with the observed average eccentricity $\bar{e} \sim 0.3$ (for planets that have not undergone tidal circularization) at the end of this migration process; (f) there is a brown dwarf desert in the mass distribution of orbiting objects that includes stellar and substellar companions.

We favor the hypothesis whereby the exoplanets that are being discovered at present, mostly in radial velocity surveys, actually constitute two different populations that overlap in mass. The first population forms by the initial assembly of a core of rock and ice, with subsequent gas accretion from a disk, and is found more frequently in high metallicity stars. The second population forms by direct collapse of gas, and its abundance is independent of stellar metallicity. Under the additional hypothesis that this second population forms by fragmentation before the gas cloud becomes opaque (rather than from gravitational instability in a rapidly rotating, highly opaque pre-stellar cloud), we suggest that the opacity limit is a natural lower limit to the mass of this class of objects, and that all the observed high-mass planets within $5 \mathrm{AU}$ of solar-type stars have migrated inwards from very large orbits. The need for this large radial interval of migration might then explain the presence of the brown dwarf desert (if massive brown dwarfs are generally not able to migrate as much because of the limited angular momentum of the circumstellar disk), and the lower eccentricities of the first population of planets (some of which would have migrated over small radial intervals and remained close to their initial circular orbits). Finally, the similarity in the eccentricity distributions of spectroscopic binaries does not seem to have any clear relation to this hypothesis.

Acknowledgements. Eduard Masana is gratefully acknowledged for providing a code to perform Kolmogorov-Smirnov tests. We are grateful to Michael Norman and Scott Tremaine for insightful discussions. I.R. acknowledges support from the Spanish MEC through a Ramón y Cajal fellowship and project AYA2006-15623-C02. J.M. is supported by the Spanish MEC project AYA2005-09413-C02-01 with EC-FEDER funding and research project 2005 SGR00728 from Generalitat de Catalunya. J.M. thanks the Institute for Advanced Study in Princeton for their hospitality when this work was being done.

\section{References}

Abt, H. A. 2005, ApJ, 629, 507

Beaulieu, J.-P., Bennett, D. P., Fouqué, P., et al. 2006, Nature, 439, 437

Black, D. C. 1997, ApJ, 490, L171

Butler, R. P., \& Marcy, G. W. 1996, ApJ, 464, L153 
Butler, R. P., Marcy, G. W., Williams, E., Hauser, H., \& Shirts, P. 1997, ApJ, 474, L115

Butler, R. P., Wright, J. T., Marcy, G. W., et al. 2006, ApJ, 646, 505

Chiang, E. I., Fischer, D., \& Thommes, E. 2002, ApJ, 565, L105

Close, L. M., Siegler, N., Freed, M., \& Biller, B. 2003, ApJ, 587, 407

Cumming, A. 2004, MNRAS, 354, 1165

Endl, M., Hatzes, A. P., Cochran, W. D., et al. 2004, ApJ, 611, 1121

Fischer, D., \& Valenti, J. 2005, ApJ, 622, 1102

Ford, E. B., Lystad, V., \& Rasio, F. A. 2005, Nature, 434, 873

Galland, F., Lagrange, A. M., Udry, S., et al. 2006, A\&A, 452, 709

González-García, B. M., Zapatero Osorio, M. R., Béjar, V. J. S., et al. 2006, A\&A, 460, 799

Gould, A., Udalski, A., An, D., et al. 2006, ApJ, 644, L37

Halbwachs, J. L., Arenou, F., Mayor, M., Udry, S., \& Queloz, D. 2000, A\&A, 355,581

Halbwachs, J. L., Mayor, M., \& Udry, S. 2005, A\&A, 431, 1129

Heacox, W. D. 1999, ApJ, 526, 928

Holman, M., Touma, J., \& Tremaine, S. 1997, Nature, 386, 254

Jones, H. R. A., Butler, R. P., Tinney, C. G., et al. 2006, MNRAS, 369, 249

Kley, W., \& Dirksen, G. 2006, A\&A, 447, 369

Kozai, Y. 1962, AJ, 67, 591

Lyo, A.-R., Song, I., Lawson, W. A., Bessell, M. S., \& Zuckerman, B. 2006, MNRAS, 368, 1451

Marcy, G. W., \& Butler, R. P. 1996, ApJ, 464, L147

Marcy, G., Butler, R. P., Fischer, D., et al. 2005, Progr. Theor. Phys. Suppl., 158, 24

Mayor, M., \& Queloz, D. 1995, Nature, 378, 355
Mayor, M., Queloz, D., \& Udry, S. 1998, in Brown Dwarfs and Extrasolar Planets, ed. R. Rebolo, E. L. Martín, \& M. R. Zapatero Osorio, ASP Conf. Ser., 134,140

McCaughrean, M. J., Close, L. M., Scholz, R.-D., et al. 2004, A\&A, 413, 1029

Nordström, B., Mayor, M., Andersen, J., et al. 2004, A\&A, 418, 989

Papaloizou, J. C. B., \& Terquem, C. 2001, MNRAS, 325, 221

Papaloizou, J. C. B., \& Terquem, C. 2006, Rept. Prog. Phys., 69, 119

Pinsonneault, M. H., \& Stanek, K. Z. 2006, ApJ, 639, L67

Pinsonneault, M. H., DePoy, D. L., \& Coffee, M. 2001, ApJ, 556, L59

Pollack, J. B., Hubickyj, O., Bodenheimer, P., et al. 1996, Icarus, 124, 62

Pourbaix, D., Tokovinin, A. A., Batten, A. H., et al. 2004, A\&A, 424, 727

Press, W. H., Teukolsky, S. A., Vetterling, W. T., \& Flannery, B. P. 1992, Numerical Recipes in Fortran 77 (Cambridge University Press)

Rees, M. J. 1976, MNRAS, 176, 483

Stepinski, T. F., \& Black, D. C. 2000, A\&A, 356, 903

Stepinski, T. F., \& Black, D. C. 2001, A\&A, 371, 250

Tabachnik, S., \& Tremaine, S. 2002, MNRAS, 335, 151

Takeda, G., \& Rasio, F. A. 2005, ApJ, 627, 1001

Tohline, J. E. 2002, ARA\&A, 40, 349

Tremaine, S., \& Zakamska, N. L. 2004, in The Search for Other Worlds, AIP Conf. Proc., 713, 243

Udry, S., Mayor, M., Naef, D., Pepe, F., et al. 2002, A\&A, 390, 267

Valenti, J. A., \& Fischer, D. A. 2005, ApJS, 159, 141

Vogt, S. S., Butler, R. P., Marcy, G. W., et al. 2002, ApJ, 568, 362

Whitworth, A., Bate, M. R., Nordlund, A., Reipurth, B., \& Zinnecker, H. 2006, in Protostars and Planets V, in press [arXiv: astro-ph/0602367] 